\title{
ANALYSIS ON DEEP LEARNING METHODS FOR ECG BASED CARDIOVASCULAR DISEASE PREDICTION
}

\author{
KUSUMA S.*AND DIVYA UDAYAN J.†
}

\begin{abstract}
The cardiovascular related diseases can however be controlled through earlier detection as well as risk evaluation and prediction. In this paper the application of deep learning methods for CVD diagnosis using ECG is addressed and also discussed the deep learning with Python. A detailed analysis of related articles has been conducted. The results indicate that convolutional neural networks are the most widely used deep learning technique in the CVD diagnosis. This research paper looks into the advantages of deep learning approaches that can be brought by developing a framework that can enhance prediction of heart related diseases using ECG.
\end{abstract}

Key words: Deep learning, Python, CVD, ECG.

AMS subject classifications. $68 \mathrm{~T} 05$

1. Introduction. According to statistics by the World Health Organization, each year 17.9 million deaths occur worldwide, all as a result of Cardiovascular Diseases [1]. Being a major concern requiring urgent attention, numerous methods and researches have been carried out in order to establish the most favorable approaches in this very crucial area of medicine. Most of these cardiovascular related diseases can however be controlled through earlier detection as well as risk evaluation and prediction. As the health systems revolve, the amount of data generated from the field is expanding vigorously. The data obtained is then used to derive more knowledge in the field as well as improvise different and innovative approaches necessary for the improvement of health services provided.

In what can be termed as the information age, research on the methods that can be used in the timely diagnosis are extensive. Forecasting is important because it eases the control of the rate and probability of someone suffering from the disease and at the same time makes it possible to reinforce the necessary measures in order to control the disease. Risk prediction of heart diseases is important because it aids in decision making on lifestyle choices and the pros and cons of some habits such as smoking, obesity and alcohol consumption. All depending on the vulnerability of the individual in getting a heart disease. Early diagnosis on the other hand makes treatment cheap which can reduce the complications as well as to reduce the cost of treatment. The revolution of the healthcare systems is very rapid. For a long time, scientists have improvised automated methods which have made forecasting of the risk easy and more accurate. Many researchers developed deep learning algorithms for ECG arrhythmia classification [2-9]. The computaion efficiency and speed achieved through convolutional neural netowrk for an individual patient [10].

In the field of medicine An electrocardiogram (ECG) is one of the most recognized and painless test that detects electrical activity in the heart using sensors that are placed on the skin over the chest [11]. Mainly there are three main parts to an ECG as in the Fig 1.1. The depolarization of the atria constitutes $\mathrm{P}$ wave and it is a small semi circular shape; Depolarization of the ventricles represents the QRS complex; and Repolarization of the ventricles constitutes the $\mathrm{T}$ wave. The rhythm of the heart in terms of beats per minute (bpm) can be easily calculated by counting the R peaks of the ECG wave during one minute of recording [12].

By taking a pulse into consideration heart rate can be calculated.If any issues realted to heart damage,size of the heart chambers or abnormal heart beat then ECG is suggested. Heart rate calculation can be done in following ways as per the article[13]:

\footnotetext{
* School of Computer Science and Engineering, Vellore Institute of technology, VIT Vellore, India.

†School of Information Technology and Engineering, Vellore Institute of technology, VIT Vellore, India
} 


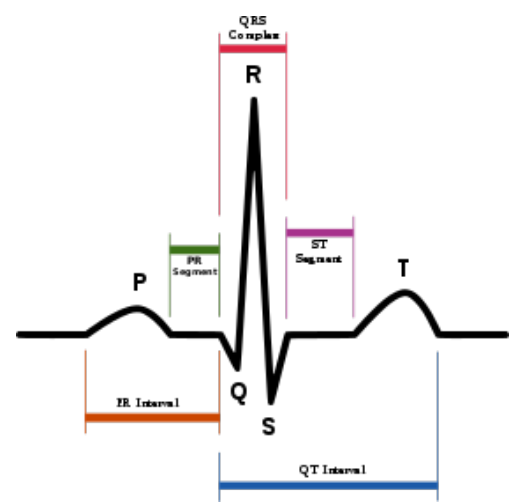

FIG. 1.1. ECG Wave

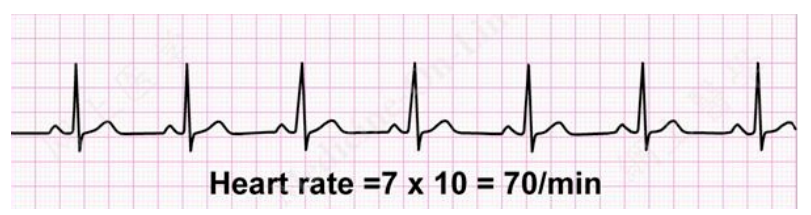

FIG. 1.2. QRS Complex

1. Make use of distance across QRS complexes to calculate the heart rate

ECG is to assess the rhythm and heart rate ,normally consists of small and large squares.for reference usually large squares are used and in each large square 5 little squares are placed.The QRS complex is the tallest part on ECG.when one QRS complex transpire,it is an indication of one heart beat has taken place. For example, let us take into 3.2 number of big squares seperates adjacent QRS cpmlexes.To determine heart rate the following calculation is used:300/3.2=93.75, which is normal as in table 1.1.

2. Second method.

30 large squares on an ECG trace represents exactly 6 seconds, multiply by 10 i.e. 6 seconds $\times 10=60$ seconds will give the number of heart beats occurred in one minute called beats per minute(BPM). For example, 7 beats counted in the period of 6 seconds then heart rate calculation is $7 \times 10=70 \mathrm{BPM}$ as shown in Fig. 1.2.

Atrial fibrillation is the common abnormal rhythm, replaces the normal heartbeat with an erratic pattern. Bradycardias is the Slow heart rhythms such as tachy-brady syndrome,atrioventricular (AV) heart block and bundle branch block. Tachycardia is a fast heart rhythm and includes:

- ventricular tachycardia (VT)

- atrial fibrillation $(\mathrm{AF})$

- supraventricular tachycardia (SVT)

- inappropriate sinus tachycardia

- atrial flutter

- ventricular fibrillation (VF).

Table 1.1 represents the normal resting heart rates at different ages according to the United States National Institutes of Health (NIH) [14].

Deep learning is a concept-oriented feature in the IT. It is instrumental in data prediction. The major obstacle is encountered when choosing the right prediction tool with the highest precision. Risk prediction has in numerous occasions been done manually. The process is however not so smooth due to several contributing risk factors that come with the disease. These factors include diabetes, hypertension and abnormal cholesterol levels. In most cases, researchers within the field of deep learning are focused on developing new data analysis methods. Not much has been done to determine the factors that affect the analysis and prediction of the results.

Deep learning methods have a wide application in the medical field. In this case, medical diagnosis is 
TABLE 1.1

Results of individual articles of deep learning and ECG based Cardiovascular disease prediction

\begin{tabular}{|l|l|}
\hline Age & Normal heart rate(BPM) \\
\hline Upto 1 month & 70 to 190 \\
\hline From 1 to 11 months & 80 to 160 \\
\hline From 1 to 2 Years & 80 to 130 \\
\hline From 3 to 4 Years & 80 to 120 \\
\hline From 5 to 6 Years & 75 to 115 \\
\hline From 7 to 9 Years & 70 to 110 \\
\hline 10 Years and above & 60 to 100 \\
\hline
\end{tabular}

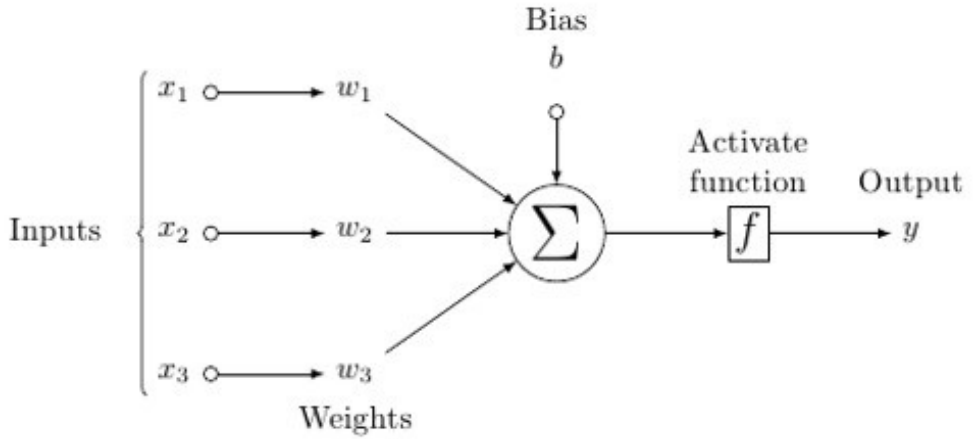

Fig. 2.1. Artificial Neuron

conducted through use-cases of deep learning networks. These include detection, segmentation, classification, prediction and other. Based on the results of the reviewed studies indicate that deep learning methods can be far superior in comparison to other high-performing algorithms. Therefore, it is safe to assume that deep learning is and will continue to diversify its uses [15].

Some of the sample public datasets features related to ECG signal consists of Age, Sex, Resting blood pressure (RBP), Cholesterol levels in the serum, chest-pain type, ST depression, Thal, Blood sugar, Maximum heartbeat rate achieved and risk levels of heart disease.

This paper is organized into three sections. First, an overview of Deep Learning and Python, next a description of research methodology, then review of deep learning in CVD diagnosis; finally, discussion on results, conclusions and future directions for development.

2. Deep learning and Python. Artificial Intelligence (AI) is a technique that emphasizes the development of intelligence machines, thinking and working like human cognitive behaviour. The model of an artificial neuron which takes an input, processes it, passed it through an activation function like the Sigmoid, return the activated output as in the Fig. 2.1.

Deep learning or deep neural network (DNN) is a subset of machine learning in Artificial intelligence that uses multiple layers for feature extraction from the input layers as represented in Fig. 2.2. The deep learning is so important because of its effectiveness on huge amount of data and ability to process large numbers of features when dealing with unstructured data. DNN achieved better results in some fields such as bioinformatics, computer vision, natural language processing( NLP), machine translation, speech and audio recognition.

The objective of a neural network is to get inputs, then to perform calculations on and to produce output to solve real world challenging problems like time series, classification of ECG signals etc. For image or object recognition Convolutional neural networks produces good results. The popular deep learning algorithms are:

- Convolutional neural networks (CNN)

- Recurrent neural networks (RNN)

- Auto-Encoders (AE) 


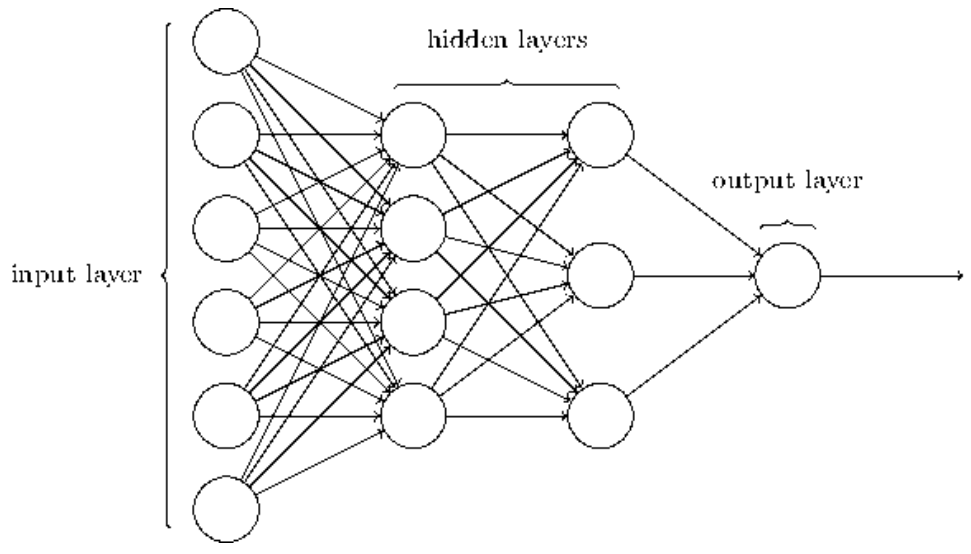

FIG. 2.2. Deep Neural Network

- Deep Boltzmann Machine (DBM)

- Long Short-Term Memory Networks (LSTMs)

- Deep Belief Networks (DBN)

The challenges for deep neural networks are computation time and overfitting. The hyper parameter such as Batching process can speed up computation process. Deep neural networks consider few parameters such as initial weights, learning rate and size to tilt overfitting some of the Regularization methods such as transfer learning, drop out, data augmentation and early stopping are applied.

Now a day's data science widely uses a popular programming language called Python to construct deep learning algorithms. To develop deep learning models for prediction, classification, understanding, perception, creation Basic knowledge towards statistics, Linear algebra, calculus, Machine learning techniques and python libraries such as Matplotlib, Numpy, Pandas, Scipy, Neural network frameworks like Keras, Tensor Flow, Theono, Pytorch is required.

Theano is a python library introduced by Yoshua Bengio for building deep neural networks to train fast on machine.It buids the model with a highly optimized solution.The libraires Tensor flow and keras as backend can be used with theono to expand the functionality.

Tensorflow is a Googles python library based on computational graphs applied for deep learning commercial grade applications. Using TensorFlow different types of deep neural networks like Autoencoders, Recursive Neural Tensor Network (RNTN), Recurrent neural network (RNN), Restricted BoltzmannMachine (RBM),Deep Boltzmann Machine (DBM),Multilayer perceptron (MLP) etc. can be built.

Keras is a portable python library with efficient numerical computation and high level neural network application programming interface (API) which allows us to define, train, developing and evaluating models of neural network with minimal lines of code.

Scikit-image is used for image processing and for statistical algorithms and data exploration stats models can be used.PyTorch is another open source library for machine learning mainy used for natural language processing and computer vision.It also includes a feature of deep neural networks built on a computational differentiation system.

Biosppy is a biosignal processing in python used for various biosignals such as Respiration, Electrocardiogram (ECG), Blood Volume Pulse (BVP), Electro Dermal Activity (EDA), Electroencephalography (EEG), Electromyography (EMG) for filtering and frequency analysis [16].

Deep Learning (DL) with python has the potential to change the future on medical imaging and diagnosis in acheiving good results comparitively with the other traditional methods.

The general research methodolgy for deep learning in health care for disease detection and prediction includes the following Phases as represented in Fig 2.3:

Phase 1: Understanding dataset is the imain step during the imception phase to avoid the problems in the development stage. 


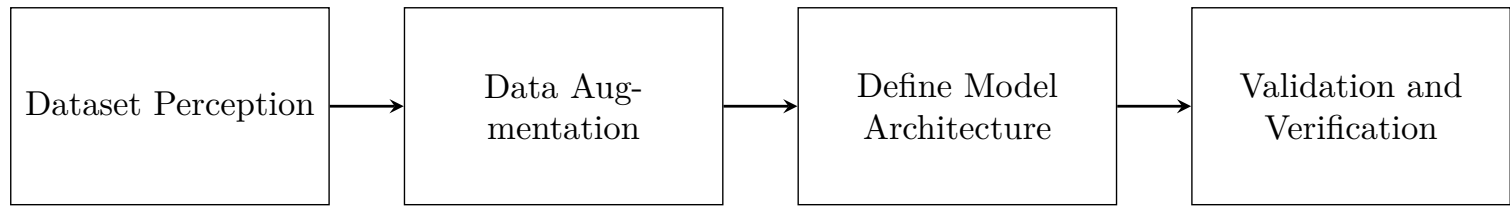

FIG. 2.3. Research Methodology.

Phase 2: Data augmentation techniques helps the researchers for training models for diverse datasets specifically for images such as cropping, padding and flipping to train large neural networks.

Phase 3: Define any new model architecture suitable for problem statement or can use already available architectures like VGG, ResNet, NASNet, UNet etc.

Phase 4: Finally validate and verify result.

\section{Method.}

3.1. Review Process. The review process is done according to the PRISMA flow diagram and protocol [17]. The diagram divided into four sections as shown in Fig 3.1. Firstly, identification of articles in the related field, followed by eliminating the duplicate articles in the screening process. Then the eligible articles are considered for review. Finally, selected articles are considered for the study.

3.2. Literature sources and process of data collection. In order to identify the results, we used the keywords Deep learning and Cardiovascular disease, Deep learning and ECG. Mainly two literature sources were used namely, IEEE digital library, PubMed [18] and dblp computer science bibliography [19] from 2016 to till date. The articles from the mentioned databases are analyzed to get the information related to the deep learning methods for cardiovascular disease prediction. We undergone the three stages to collect the data from the sources.

Stage 1: Searching articles related to the keywords specified above.

Stage 2: Analysed the literature and excluded which are not related to research.

Stage 3: Finally at the result section presented the qualitative data such as type of data sets, deep learning methods.

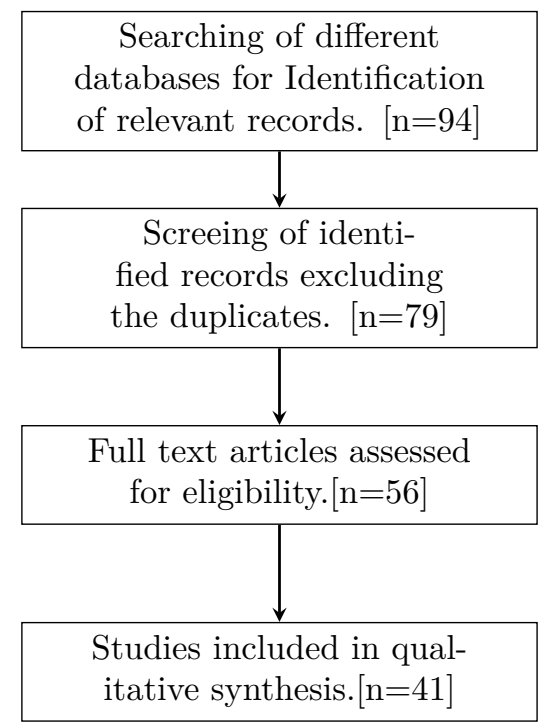

FIG. 3.1. Review process flow diagram 
4. Review Results. The performance analysis is done over several state-of-the-art models through the Type I measures such as positive measures like Accuracy, Sensitivity and Specificity. The main terms considered patient as positive sign for disease and healthy person as negative sign for disease [18].

Accuracy is to test its ability to correctly dive into the samples of healthy persons and patients. This can be stated mathematically as:

$$
\text { Accuracy }=(T P+T N) /(T P+T N+F P+F N)
$$

Sensitivity test is to determine the patient samples correctly through the proportion of true positive in patient samples. This can be stated mathematically as:

$$
\text { Sensitivity }=T P /(T P+F N)
$$

Specificity test is to determine the healthy samples correctly through the proportion of true negative in healthy samples. This can be stated mathematically as:

$$
\text { Specificity }=T N /(T N+F P)
$$

where FP stand for "false positive" which indicates the number of samples incorrectly identified as patient; $F N$ for "false negative" which indicates the number of samples incorrectly identified as heallthy. TP for "true positive" which indicates the number of samples correctly identified as patient; $T N$ for "true negative" indicates the number of samples correctly identified as healthy.

5. Proposed methodology. The proposed model for heart disease prediction using ECG signals includes three major phases: Pre-processing, Feature Extraction, and Classification. In the first phase, both filtering process and heart beat detection takes place. After pre-processing, feature extraction will be carried out, from which the optimal features will be selected using a new Algorithm. Subsequently, the optimal features will be subjected to classification process, where the Optimized Neural Network (NN) will be used to predict the presence of heart disease. Further, to make the prediction model more accurate, the training of NN will be done using the proposed algorithm via selecting the optimal weight.Moreover, the proposed improved algorithm deals with the adaptive mutation process as the conventional algorithm concentrates only on the static mutation process [42]. The adaptiveness in proposed algorithm ens ures the optimal tuning more appropriate. The overall architecture of the proposed scheme is demonstrated by Fig. 5.1.

The proposed heart disease prediction system using ECG signals will be implemented in Python, and the experimental investigation will be carried out. The performance analysis will be done by comparing the proposed model over several state-of-the-art models through the Type 1 measures and Type 2 measures. Here, Type I measures are positive measures like Accuracy, Sensitivity, Specificity, Precision, Negative Predictive Value (NPV), F1Score and Mathews correlation coefficient (MCC), and Type II measures are negative measures like False positive rate (FPR), False negative rate (FNR), and False Discovery Rate (FDR).

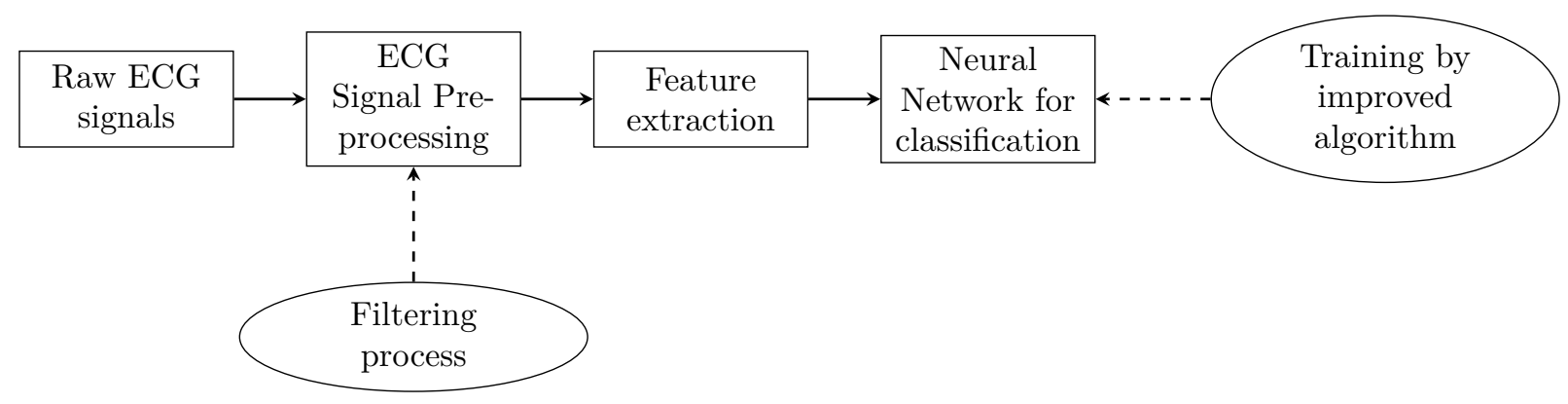

FIG. 5.1. Overall Architecture of the Proposed Heart Disease Prediction Model. 
TABLE 4.1

Results of individual articles of deep learning and ECG based Cardiovascular disease prediction

\begin{tabular}{|c|c|c|c|}
\hline Ref & Method & Dataset/Datasource & Outcome \\
\hline [19] & 1D-CNN & $\begin{array}{l}\text { MIT-BIH, St.-Petersberg, PTB } \\
\text { databases }\end{array}$ & $\begin{array}{l}\text { Achieved accuracy of } 97.70 \text {, sensitivity } 99.71 \text {, and } \\
\text { specificity } 98.24 \text { respectively. }\end{array}$ \\
\hline [20] & LenET-5T CNN & $\begin{array}{l}\text { Physionet Apnea ECG dataset, } \\
\text { UCD dataset }\end{array}$ & $\begin{array}{l}\text { Achieved accuracy of } 97.1 \text {, sensitivity } 100 \text {, and speci- } \\
\text { ficity } 91.7 \text {, respectively. }\end{array}$ \\
\hline [21] & $\begin{array}{l}\text { DL-CCANet and } \\
\text { TL-CCANet }\end{array}$ & $\begin{array}{l}\text { MIT-BIH database, INCART } \\
\text { database }\end{array}$ & $\begin{array}{l}\text { DL-CCANet, achieved accuracies of } 95.25 \text { and } 94.0 \text {, the } \\
\text { TL-CCANet achieved the highest accuracy of } 95.52 \text {. }\end{array}$ \\
\hline$[22]$ & $\begin{array}{l}\text { DNN (Deep Convo- } \\
\text { lutional neural net- } \\
\text { work) }\end{array}$ & 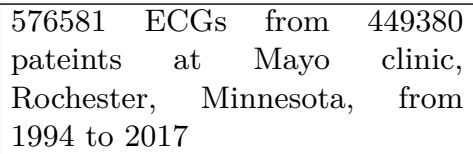 & Obtained accuracy of 90 \\
\hline [23] & $\begin{array}{l}\text { Deep Learning algo- } \\
\text { rithm }\end{array}$ & $\begin{array}{l}\text { Mayo clinic ECG laboratory in } \\
\text { September } 2018\end{array}$ & $\begin{array}{l}\text { Achieved accuracy of } 86.5 \text {, sensitivity } 82.5 \text {, and speci- } \\
\text { ficity } 86.8 \text {, respectively. }\end{array}$ \\
\hline$[24]$ & $\begin{array}{l}\text { Multilayer percep- } \\
\text { tron and CNN }\end{array}$ & physiobank.com, Kaggle.com & Achieved accuracy of 88.7 for MLP and 83.5 for CNN. \\
\hline [25] & $\begin{array}{l}\text { Linear support vec- } \\
\text { tor machine (SVM) }\end{array}$ & MIT-BIH arrhythmia database & Achieved accuracy of 97.77 and 97.08 . \\
\hline [26] & CNN & PhysioNet database & $\begin{array}{l}\text { The performance on overall F1 score reached to } 0.82 \\
\text { when algorithm applied. }\end{array}$ \\
\hline$[27]$ & CNN & PhysioNet database & $\begin{array}{l}\text { The rhythm classification performance reached an } \\
\text { overall F } 1 \text { score of } 0.82 \text { when applying the algorithm } \\
\text { to the hidden test set. }\end{array}$ \\
\hline [28] & $\begin{array}{l}\text { Deep convolutional } \\
\text { neural network }\end{array}$ & MIT - BIH Arrhythmia database & $\begin{array}{l}\text { Deep 1D-CNN achieved a recognition overall accuracy } \\
\text { of } 17 \text { cardiac arrhythmia disorders (classes) at a level } \\
\text { of } 91.33 \text { and classification time per single sample of } \\
0.015 \text {.s }\end{array}$ \\
\hline [29] & $\begin{array}{l}\text { Convolutional neural } \\
\text { network }(\mathrm{CNN})\end{array}$ & Publicly available database & $\begin{array}{l}\text { Accuracy of } 94.03 \text { and } 93.47 \text { with and without noise } \\
\text { removal respectively. }\end{array}$ \\
\hline [30] & $\begin{array}{l}\text { CNN-BLSTM net- } \\
\text { work model }\end{array}$ & MIT - BIH Arrhythmia database & $\begin{array}{l}\text { Achieved an accuracy o } 96.59 \text {, a sensitivity of } 99.93 \text { and } \\
\text { a specificity of } 97.03\end{array}$ \\
\hline [31] & $\begin{array}{l}\text { Deep neural network } \\
(\mathrm{DNN})\end{array}$ & $\begin{array}{l}\text { Physio net data and Novel } \\
\text { dataset of } 328 \text { records from } 328 \\
\text { unique patients }\end{array}$ & $\begin{array}{l}\text { Rhythm classes were examined and had higher sensi- } \\
\text { tivity of } 94.1 \text { than the averaged cardiologists of } 78.4 \text {. }\end{array}$ \\
\hline$[32]$ & $\begin{array}{l}\text { LSTM based auto- } \\
\text { encoder network } \\
\text { with SVM (support } \\
\text { vector machine) }\end{array}$ & MIT-BIH arrhythmia database & $\begin{array}{l}\text { This method achieved average accuracy of } 99.45,98.63 \\
\text { sensitivity, and } 99.66 \text { specificity in the beat-based } \\
\text { cross-validation approach. }\end{array}$ \\
\hline [33] & CNN and LSTM & MIT-BIH arrhythmia database & $\begin{array}{l}\text { Accuracy of } 99.26 \text { and } 94.20 \text { were achieved under class- } \\
\text { oriented scheme and subject-oriented scheme. }\end{array}$ \\
\hline$[34]$ & $\mathrm{CNN}$ & MIT-BIH arrhythmia database & $\begin{array}{l}\text { As a result, classified the ECG beats into five categories } \\
\text { with an average accuracy of } 99.21 \text {. }\end{array}$ \\
\hline$[35]$ & $\begin{array}{l}\text { Deep residual convo- } \\
\text { lutional neural net- } \\
\text { work (DR-CNN), }\end{array}$ & MIT-BIH arrhythmia database & $\begin{array}{l}\text { Achieved the average detection accuracy of } 99.0349 \text {, } \\
99.4980 \text { and } 99.3347 \text { for multiclass classification, ven- } \\
\text { tricular ectopic beat (Veb) and supra-Veb (Sveb) recog- } \\
\text { nition, respectively. }\end{array}$ \\
\hline$[36]$ & $\begin{array}{l}\text { Deep multi-task } \\
\text { learning approach }\end{array}$ & MIT-BIH arrhythmia database & $\begin{array}{l}\text { Improved the accuracy of ECG data analysis by up to } \\
\text { about } 5.1\end{array}$ \\
\hline$[37]$ & $\begin{array}{l}\text { Extreme Learning } \\
\text { Machine(ELM) }\end{array}$ & MIT-BIH arrhythmia database & $\begin{array}{l}\text { This paper has a } 1.7 \text { increase, and the sensitivity of the } \\
\mathrm{N}, \mathrm{S}, \mathrm{V} \text {, and Q categories are } 99.12,75.2,93.72 \text {, and } \\
98.64 \text {, respectively. }\end{array}$ \\
\hline
\end{tabular}


TABLE 4.2

Results of individual articles of deep learning and ECG based Cardiovascular disease prediction (continuation)

\begin{tabular}{|l|l|l|l|}
\hline Ref & Method & Dataset/Datasource & Outcome \\
\hline$[38]$ & Transfer learning & $\begin{array}{l}\text { Physio net/CinC Challenge 2017 } \\
\text { dataset }\end{array}$ & The method achieved F1F1 score of 0.89 and 0.86. \\
\hline$[39]$ & $\begin{array}{l}\text { LSTM deep neural } \\
\text { network }\end{array}$ & $\begin{array}{l}400,000 \text { clean PPG (photo } \\
\text { plethy smography) samples to } \\
\text { learn typical PPG morphology } \\
\text { and rhythm }\end{array}$ & $\begin{array}{l}\text { Tcardiac abnormality recognition approach recognises } \\
60+\text { of ECG-detected PVCs in PPG signal, with a } \\
\text { false positive rate of 23 - demonstrating the compelling } \\
\text { power and value of this novel approach. }\end{array}$ \\
\hline$[40]$ & RNN & $\begin{array}{l}\text { Physio net/CinC Challenge 2017 } \\
\text { dataset }\end{array}$ & Achieved the maximum accuracy of 79 \\
\hline$[41]$ & CNN & MIT-BIH arrhythmia database & Accuracy obtained 99,97.6 \\
\hline
\end{tabular}

6. Discussion and Conclusion. Heart related illnesses and other cardiovascular diseases are a menace in the society and if they are not eradicated can cause drastic outcomes in the long run. Considering the mortality rate of the patients, this calls for the disease to be a major concern. Manually establishing the chances of getting the disease is very difficult and highly likely to be ineffective. However, deep learning techniques come in handy to solve the problem by evaluating the risk factors using the data presented in order to generate a forecast on the odds of as the output.

The objective of this paper was to study research papers in the field of deep learning methods on ECG based cardiovascular disease prediction. The available public datasets of ECG such as MIT-BIH, INCART, Physionet and Kaggle database. Despite the field of medicine being vast and generating a lot of data every single second, the infrastructure is set in place to store all the data and make it secure and readily available. From the findings from this research it is conclusive to say that Conventional Neural Network which is a method of deep learning has a high accuracy level on large datasets.Python is playing a major role in implementation of deep learning models. This may reduce the risk of late diagnosis by earlier prediction and improves the quality in health care.Deep learning methods in medical diagnosis will not replace practitioners, the devlopment of DL models is to help doctors in early decision making.

7. Limitations and Future Research. Development of deep learning is still underdeveloped. There is still not enough research on the theories and algorithm for use in risk prediction. The accuracy and efficiency of the data mining methods used may be compromised especially when the wrong model is used or when handling large data capacities. There being enough research on complex data, the research may be altered by the gap in knowledge considering the several colliding explanations.It is expensive to build an expert heart disease prediction system.

Having the necessary deep learning tool models is very effective in forecasting. However, it does not really solve the problem at real time. Instead, the use of wearable sensors can most effectively solve the problem through giving feedback in real time. Due to the advancement of technology, the exposure to the internet and social media increases the bulk of data that is created every second. Data mining in the health industry is very essential. Data obtained could be structured, unstructured, complex or multileveled.

Smart phones have changed the health sector due to innovations such as medical apps. Online prescriptions, assessment, clinical decision support, treatment practice management, and self-care all are simplified. Smart watches that can keep track of various body activities such as pulse rate, temperature and continuous health management by using attached physiological sensors. Present deep learning tools rely on assumptions that variables are related, there is still a research gap regarding variables that are not related to each other in any particular manner. If a common model was introduced to handle all types of datasets this would be easy and more accurate. This will most efficiently make them more reliable and accurate in giving the right predictions.

The process of human learning is still a mystery and complex. It has not yet been implemented in machine learning. However, given the interest of researchers in the field of machine learning theories and algorithms, incorporation of multiple sensory modalities such as vision, sound and touch in self-supervised learning to predict one sensory experience from the others might become a reality. 


\section{REFERENCES}

[1] World Health Organization, Condiovascular Diseases. Available at https://www.who.int/health-topics/cardiovasculardiseases.

[2] G. K. Malik, Y. Kumar, and M. Panda., (2017). Cardiac arrhythmia detection in ECG signals by feature extraction and support vector machine," in Proceedings of the Second International Conference on Research in Intelligent and Computing in Engineering, ACSIS, vol. 10, pp. 241-244.

[3] E. J. D. S. Luz, W. R. Schwartz, G. CÁmara-Chávez, and D. Menott, (2016).ECG-based heartbeat classification for arrhythmia detection: a survey,"Computer Methods and Programs in Biomedicine, vol. 127, pp. 144-164, 2016.

[4] J. A. Gutiérrez-Gnecchi, R. Morfin-Magaña, D. Lorias-Espinoza Et Al., (2017)."DSP-based arrhythmia classification using wavelet transform and probabilistic neural network," Biomedical Signal Processing and Control, vol. 32, pp. 44-56.

[5] B. Pyakillya, N. Kazachenko, and N. Mikhailovsky, (2017).Deep learning for ECG classification," Journal of Physics: Conference Series, vol. 913, pp. 1-5.

[6] Isin, A., And Ozdalili, S., (2017). Cardiac arrhythmia detection using deep learning. Procedia computer science, 120, 268-275.

77] B.M. M. A. Rahhal, Y. Bazi, H. AlHichri, N. Alajlan, F. Melgani, and R. R. Yage, (2016).Deep learning approach for active classification of electrocardiogram signals," Information Sciences, vol. 345, pp. 340-354.

[8] Rangayyan, R. M., (1999). Biomedical Signal Analysis: A Case-Study Approach. IEEE Press.

[9] Bakator, M., and Radosav, D., (2018). Deep learning and medical diagnosis: A review of literature. Multimodal Technologies and Interaction, 2(3), 47.

[10] S. Kiranyaz, T. Ince, and M. Gabbouj , (2016).Real-time patientspecific ECG classification by 1-D convolutional neural networks,IEEE Trans. Biomed. Eng., vol. 63, no. 3, pp. 664-675.

[11] Electrocardiography. Available https://en.wikipedia.org/wiki/Electrocardiography.

[12] How to calculate Heart Rate from ECG. Available at https://www.wikihow.com/Calculate-Heart-Rate-from-ECG.

[13] What should my heart rate be? Available at https://www.medicalnewstoday.com/articles/235710.php

[14] PubMed, Available at https://www.ncbi.nlm.nih.gov/pubmed.

[15] DBLP search on "deep learning and cardiovascular". Available at https://dblp.uni-trier.de/

[16] Carreiras C, Alves AP, Lourenço A, Canento F, Silva H, Fred A, et al. , BioSPPy - Biosignal Processing in Python, 2015-, https://github.com/PIA-Group/BioSPPy/

[17] Moher, D.; Liberati, A.; Tetzlaff, J.; Altman, D.G, Prisma Group. Preferred reporting items for systematic reviews and meta-analyses: The PRISMA statement. Int. J. Surg. 2010, 8, 336-341. [CrossRef] [PubMed]

[18] Baratloo, A., Hosseini, M., Negida, A., and El Ashal, G. , (2015). Part 1: simple definition and calculation of accuracy, sensitivity and specificity.

[19] Hasan, N. I., and Bhattacharjee, (2019). Deep Learning Approach to Cardiovascular Disease Classification Employing Modified ECG Signal from Empirical Mode Decomposition. Biomedical Signal Processing and Control, 52, 128-140.

[20] Wang, T., Lu, C., Shen, G., And Hong, F, (2019). Sleep apnea detection from a single-lead ECG signal with automatic feature-extraction through a modified LeNet-5 convolutional neural network. PeerJ, 7, e7731.

[21] Yang, W., Si, Y., Wang, D., And Zhang, G., (2019). A Novel Approach for Multi-Lead ECG Classification Using DLCCANet and TL-CCANet. Sensors, 19(14), 3214.

[22] Galloway, C. D., Valys, A. V., Shreibati, J. B., Treiman, D. L., Petterson, F. L., Gundotra, V. P., ... and Ackerman, M. J., (2019). Development and Validation of a Deep-Learning Model to Screen for Hyperkalemia From the Electrocardiogram. JAMA cardiology, 4(5), 428-436.

[23] Attia, Z. I., Kapa, S., Yao, X., Lopez-Jimenez, F., Mohan, T. L., Pellikka, P. A., ... And Noseworthy, P. A., (2019). Prospective validation of a deep learning electrocardiogram algorithm for the detection of left ventricular systolic dysfunction. Journal of cardiovascular electrophysiology, 30(5), 668-674.

[24] Savalia, S., and Emamian, V. , (2018). Cardiac arrhythmia classification by multi-layer perceptron and convolution neural networks. Bioengineering, $5(2), 35$.

[25] Yang, W., Si, Y., Wang, D., And Guo, B., (2018). Automatic recognition of arrhythmia based on principal component analysis network and linear support vector machine. Computers in biology and medicine, 101, 22-32.

[26] Sodmann, P., Vollmer, M., Nath, N., And Kaderali, L. , (2018). A convolutional neural network for ECG annotation as the basis for classification of cardiac rhythms. Physiological measurement, 39(10), 104005.

[27] Yildirim, Ö., PŁawiak, P., Tan, R. S., And Acharya, U. R., (2018). Arrhythmia detection using deep convolutional neural network with long duration ECG signals. Computers in biology and medicine, 102, 411-420.

[28] Acharya, U. R., Oh, S. L., Hagiwara, Y., Tan, J. H., Adam, M., Gertych, A.,and San Tan, R. , (2017). A deep convolutional neural network model to classify heartbeats. Computers in biology and medicine, 89, 389-396.

[29] Dang, H., Sun, M., Zhang, G., Qi, X., Zhou, X., And Chang, Q. , (2019). A novel deep arrhythmia-diagnosis network for atrial fibrillation classification using electrocardiogram signals. IEEE Access.

[30] Hannun, A. Y., Rajpurkar, P., Haghpanahi, M., Tison, G. H., Bourn, C., Turakhia, M. P., And NG, A. Y. , (2019). Cardiologist-level arrhythmia detection and classification in ambulatory electrocardiograms using a deep neural network. Nature medicine, 25(1), 65-69.

[31] Hou, B., YAng, J., Wang, P., And YAn, R. , (2019). LSTM Based Auto-Encoder Model for ECG Arrhythmias Classification. IEEE Transactions on Instrumentation and Measurement.

[32] Shi, H., Qin, C., XiaO, D., ZhaO, L., And LiU, C. , (2019). Automated heartbeat classification based on deep neural network with multiple input layers. Knowledge-Based Systems, 105036.

[33] Ji, Y., Zhang, S., And XiaO, W. , (2019). Electrocardiogram Classification Based on Faster Regions with Convolutional 
Neural Network. Sensors, 19(11), 2558.

[34] Li, D., Zhang, H., Liu, Z., Huang, J., And Wang, T. , (2019). Deep residual convolutional neural network for recognition of electrocardiogram signal arrhythmias. Sheng wu yi xue gong cheng xue za zhi= Journal of biomedical engineering= Shengwu yixue gongchengxue zazhi, 36(2), 189-198.

[35] Imsanto, E., Ramli, K., And Sudiana, D. , (2019, July). Real-Time Classification for Cardiac Arrhythmia ECG Beat. In 2019 16th International Conference on Quality in Research (QIR): International Symposium on Electrical and Computer Engineering (pp. 1-5). IEEE.

[36] Ji, J., Chen, X., Luo, C., ANd Li, P. , (2018, March). A deep multi-task learning approach for ECG data analysis. In 2018 IEEE EMBS International Conference on Biomedical and Health Informatics (BHI) (pp. 124-127). IEEE.

[37] Zhang, X., Li, R., Liu, Y., GaO, S., Zhang, H., Shen, S., and Wang, Z. , (2018, August). Classification of Arrhythmia Based on Extreme Learning Machine. In 2018 10th International Conference on Intelligent Human-Machine Systems and Cybernetics (IHMSC) (Vol. 2, pp. 123-126). IEEE.

[38] Chen, L., Xu, G., Zhang, S., Kuang, J., and HaO, L. , (2019). Transfer Learning for Electrocardiogram Classification Under Small Dataset. In Machine Learning and Medical Engineering for Cardiovascular Health and Intravascular Imaging and Computer Assisted Stenting (pp. 45-54). Springer, Cham.

[39] Whiting, S., Moreland, S., Costello, J., Colopy, G., and McCann, C. , (2018). Recognising Cardiac Abnormalities in Wearable Device Photoplethysmography (PPG) with Deep Learning. arXiv preprint arXiv:1807.04077.

[40] B. Taji, A. D. Chan, and S. Shirmohammad , (2018).False alarm reduction in atrial fibrillation detection using deep belief networks,IEEE Trans. Instrum. Meas., vol. 67, no. 5, pp. 1124-1131.

[41] P. Schwab, G. C. Scebba, J. Zhang, M. Delai, and W. Karlen , (2017).Beat by beat: Classifying cardiac arrhythmias with recurrent neural networks, in Proc. Comput. Cardiol., vol. 44, pp. 1-4.

[42] BR RAJAKUmaR, (2013).Static and adaptive mutation techniques for genetic algorithm: a systematic comparative analysis, International Journal of Computational Science and Engineering, vol. 8, no. 2, pp. 180-193.

Edited by: Swaminathan JN

Received: Nov 27, 2019

Accepted: Jan 20, 2020 